\title{
A new coumarin from Calophyllum hosei
}

\section{Abstract}

A new coumarin, hoseimarin (1), together with four other xanthones, trapezifolizanthone (2), osajaxanthone (3), $\beta$-mangostin (4) and caloxanthone A (5), were isolated from the stem bark of Calophyllum hosei. The structures of these compounds were established by using spectroscopic analysis which included 1H NMR, 13C NMR, COSY, DEPT, HMQC and HMBC experiments.

Keyword: Calophyllum hosei; Coumarin; Hoseimarin; Trapezifolixanthone 